\title{
Surface Finish Quality of the Outer AXAF Mirror Pair Based on X-ray Measurements of the VETA-I
}

\author{
John P. Hughes, Daniel Schwartz, \\ Andrew Szentgyorgyi, Leon Van Speybroeck, and Ping Zhao \\ Harvard-Smithsonian Center for Astrophysics \\ 60 Garden Street, Cambridge, MA 02138
}

\begin{abstract}
We employ the X-ray measurements of the VETA-I taken at the X-Ray Calibration Facility (XRCF) of the Marshall Space Flight Center (MSFC) to extract information about the surface finish quality of the outermost pair of AXAF mirrors. The particular measurements we consider are one dimensional scans of the core of the point response function (PRF) (full width half maximum [FWHM] scans), the encircled energy as a function of radius, and one dimensional scans of the wings of the PRF. We discuss briefly our raytrace model which incorporates the numerous effects present in the VETA-I test, such as the finite source distance, the size and shape of the X-ray source, the residual gravitational distortions of the optic, the despace of the VETA-I, and particulate contamination. We show how the data constrain the amplitude of mirror surface deviations for spatial frequencies greater than about $0.1 \mathrm{~mm}^{-1}$. Constraints on the average amplitude of circumferential slope errors are derived as well.
\end{abstract}

\section{INTRODUCTION}

One of the principal goals of the Advanced X-ray Astrophysics Facility (AXAF) is to perform sensitive, high spatial resolution imaging over a broad $\mathrm{X}$-ray bandwidth. This capability is provided in large part by the High Resolution Mirror Assembly (HRMA), which consists of a set of nested Wolter Type-I mirrors. In the summer of 1991, the Verification Engineering Test Article (VETA-I), consisting of the outermost pair of HRMA shells (P1/H1) assembled into a mounting fixture, was delivered to the XRCF for X-ray testing. The main purpose of the test was to demonstrate FWHM imaging performance of better than $0.5^{\prime \prime}$ for the optic. An additional secondary goal was verification of the optical surface metrology. Since schedule pressures made it impossible to obtain complete final metrology of the polished surfaces, it was decided to use the X-ray data itself to estimate the surface finish quality of P1/H1.

We consider three types of data in this paper: (1) one-dimensional scans of the core of the PRF at the single X-ray energy of $1.488 \mathrm{keV} ;(2)$ encircled energy as a function of radius for three X-ray energies: 0.277 , 1.488 , and $2.067 \mathrm{keV}$; and (3) one dimensional large angle scans of the wings of the PRF for five X-ray test energies: $0.277,0.932,1.488,2.067$, and $2.334 \mathrm{keV}$. As we show below, the first data set is most constraining of the circumferential slope errors on the surface. The second data set is most sensitive to the power spectral density (PSD) of surface irregularities over spatial frequencies of $f=0.05-24 \mathrm{~mm}^{-1}$. The last data set is sensitive to the amount of particulate contamination on the surface in addition to the PSD of surface irregularities over $f=1-100 \mathrm{~mm}^{-1}$.

The shape of the VETA-I PRF in the core within a radius of about $100 \mu \approx 2^{\prime \prime}$ (we assume a focal plane scale of $0.02^{\prime \prime} / \mu$ throughout this paper) was determined largely by the test conditions. For example, the optics were not cut to their nominal flight lengths, which meant that it was impossible to space them properly. The optical elements were separated by a despace of $109.03 \mathrm{~mm}$ in addition to the nominal design spacing. The finite source distance also degraded the imaging performance in the core. In order to faithfully reproduce these and other effects in the FWHM scans and encircled energy data, it was required that a raytrace calculation be performed. On the other hand the data taken far from the core, such as the wing scan data, could be liandled analytically. 


\section{RAYTRACE MODEL}

Our calculation for the X-ray performance of the VETA-I consists of two parts: (1) the raytrace program OSAC (Optical Surface Analysis Code) written by P. Glenn, and (2) a stand-alone post-processing program developed at SAO.

OSAC incorporates the mirror surface prescription (including low order error terms, such as axial sag) and various VETA-I ground calibration effects: the finite source distance $(518160 \mathrm{~mm}$, measured from the front of the VETA-I), the despace, and gravitational distortions. The latter were implemented in OSAC as a set of Fourier-Legendre coefficients which were determined from fits to NASTRAN finite element analysis models of the VETA-I in the test configuration.

SAO's post processing software was able to include the measured intensity distribution of the XRCF $\mathrm{X}$-ray source, ${ }^{1}$ obscuration due to the aperture support struts, an arbitrary axial focus location, and circular pinholes of arbitrary size placed at arbitrary positions in the focal plane. Scattering of X-rays due to particulate contamination on the surface is also included. ${ }^{2}$

Various mirror fabrication errors: $\Delta \Delta R$, circumferential slope, and axial slope errors, were implemented in the post processing software. Values for the first error term were obtained from the mirror manufacturer, Hughes Danbury Optical Systenıs (HDOS) (P. Reid, private communication). The VETA-I test data were not very sensitive to this error term. The magnitude of circumferential slope errors was parameterized as a (one-dimensional) normally distributed quantity, and the direction of scatter was taken to be orthogonal to the axial slope direction. Our results show that the FWHM data scans were somewhat sensitive to the magnitude of this error. Axial slope errors were parameterized in terms of the PSD of the surface. Most of the results we quote in this paper are based on the assumption of a PSD which varies as a power-law in spatial frequency $f$, i.e., $2 W_{1}(f)=C f^{-p}$. We allow the amplitude $C$ and power-law index $p$ to be free parameters. By convention, the amplitude of the PSD is represented in terms of the band-width limited integral for the total surface roughness

$$
\sigma^{2}=2 \int_{f_{1}}^{f_{2}} W_{1}(f) d f
$$

We quote results for the roughness per surface using limits of $1 \mathrm{~mm}^{-1}$ and $1000 \mathrm{~mm}^{-1}$.

\section{FWHM}

The data corresponding to the full-width-half-maximum (FWHM) of the PRF of the VETA-I are shown in Fig. 1. This intensity distribution was made by stepping a $5 \mu$ diameter circular pinhole in $2 \mu$ steps through the core of the PRF, after an initial sequence of procedures demonstrated a relative tilt alignment between the optical elements of less than $0.1^{\prime \prime}$ and the location of the axial focus position to better than $25 \mu$. The data were taken with an aluminum anode target which produced characteristic $\mathbf{K} \alpha$ line emission at an (emission-weighted) energy of $1.488 \mathrm{keV}$. We extracted the total number of counts from a restricted range of pulse height $(\mathrm{PH})$ channels of the observed proportional counter spectrum. No corrections for background, deadtime, or pulse pileup were applied since the relative correction from point to point was less than a few percent for these effects.

We present a study of the VETA-I FWHM using our raytrace model with the goal of identifying the dominant contributors to the FWHM performance of the VETA-I, whether test-induced (such as the finite source distance) or intrinsic to the mirror surface itself. Table 1 shows predicted FWHM values for several raytrace models where various mirror performance errors are introduced each in turn. In all cases the calculations are based on $5 \times 10^{5}$ rays of which about 1000 pass through the central pinhole, which ensures statistical precision of about $5 \%$ in the modelled FWHM values. 

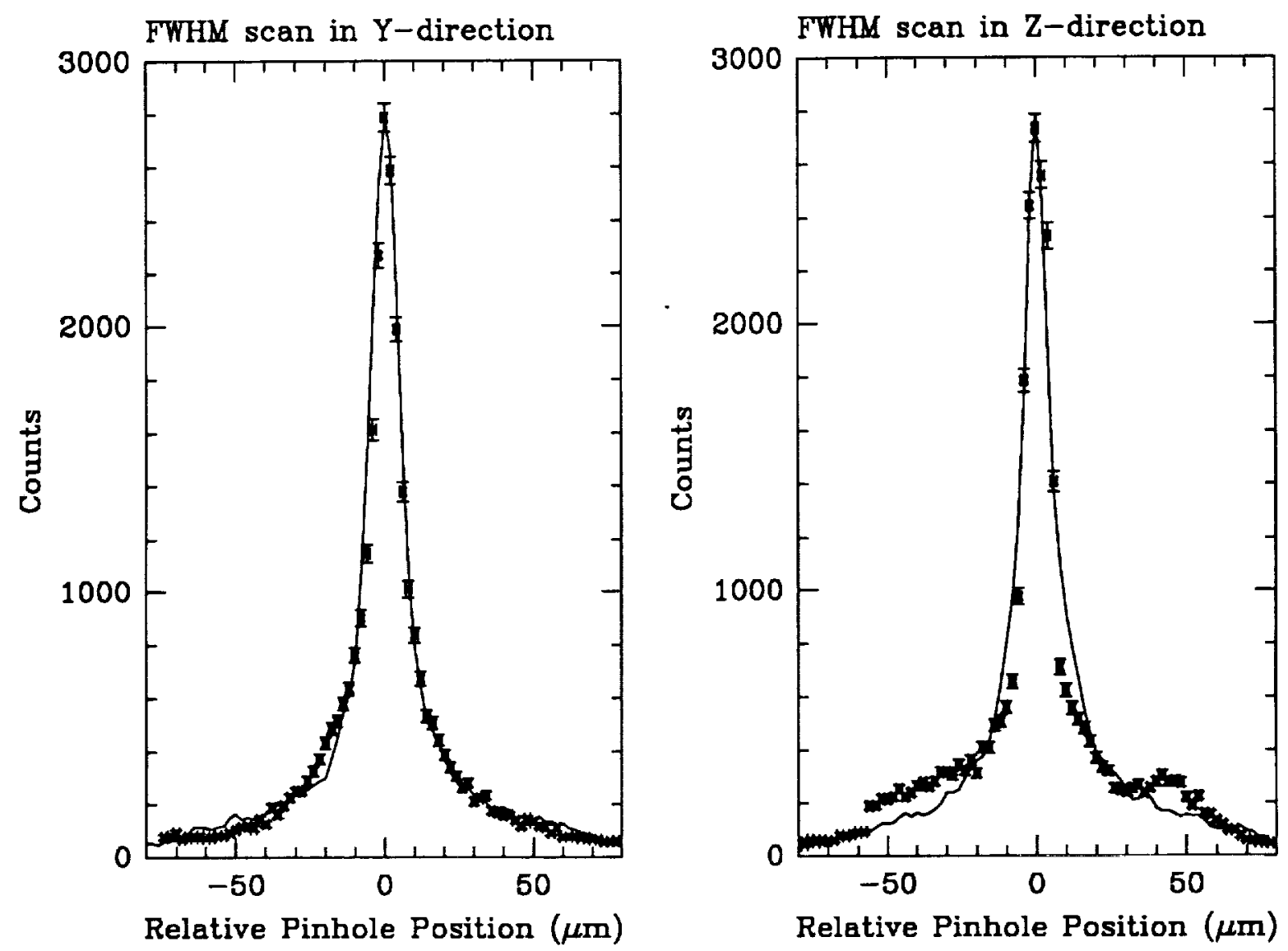

Figure 1 - The data points obtained during the FWHM scans of the core of the VETA-I point response function with a $5 \mu\left(0.1^{\prime \prime}\right)$ diameter pinhole moved in $2 \mu$ steps. The left panel shows results from scans along the $\mathrm{Y}$-direction (horizontal); while the right panel shows scans along the $\mathrm{Z}$-direction (vertical). The solid curves show the model assuming $3 \mu \mathrm{rad}$ circumferential slope errors as discussed in the text.

\begin{tabular}{|c|c|c|}
\hline \multicolumn{3}{|c|}{$\begin{array}{c}\text { Table } 1 \\
\text { Predicted VETA-I FWHM }\end{array}$} \\
\hline & $\begin{array}{c}\text { Y-FWHM } \\
(\operatorname{arcsec})\end{array}$ & $\begin{array}{l}\text { Z-FWHM } \\
(\operatorname{arcsec})\end{array}$ \\
\hline Measured Value & $0.219 \pm 0.030$ & $0.221 \pm 0.030$ \\
\hline 1. Grav., Finite Conj., Despace & 0.132 & 0.140 \\
\hline 2. Grav., Finite Conj., Despace, Source size & 0.203 & 0.203 \\
\hline 3. Case 2 plus Lev II Req axial slope errors & 0.208 & 0.206 \\
\hline 4. Case 2 plus Lev II Goal axial slope errors & 0.209 & 0.210 \\
\hline 5. Case 2 plus $\Delta \Delta R$ errors & 0.201 & 0.206 \\
\hline 6. Case 2 plus circum. slope errors & 0.326 & 0.336 \\
\hline 7. Case 2 plus axial sag & 0.202 & 0.202 \\
\hline
\end{tabular}


The first two models include no mirror degradation, and instead include only the so-called facility effects: the calculated residual gravitational distortion of the mirror, the finite source distance (finite conjugate), the mirror despace, and the finite source size. This last effect was included by inputting the actual measured intensity distribution of the XRCF X-ray source ${ }^{1}$ to the raytrace model. Comparison of the predicted values in these two cases reveals that the finite size of the source is the dominant facility induced effect to the FWHM.

The remaining five cases show how the FWHM increases (or not) when various mirror performance error terms are included. Cases 3 and 4 include the axial slope errors which correspond to the Level II requirement and goal on HRMA performance as established by the AXAF project. Case 5 includes $\Delta \Delta R$ errors as discussed above. Case 6 includes circumferential slope errors with values, determined by HDOS metrology, of $6.446 \mu \mathrm{rad}$ (on P1 after the sixth polishing cycle) and $9.745 \mu$ rad (on $\mathrm{H} 1$ after the fifth polishing cycle). The final case includes the maximal sag error (value plus quoted error), as known at the time of the test, $1585 \AA(\mathrm{P} 1)$ and $447 \AA(\mathrm{H} 1)$. Clearly the dominant mirror effect is the circumferential slope errors, for which metrology of the current optical surfaces (after 7 cycles of polishing) does not yet exist.

Given the large disparity between the predictions of the current raytrace model and the actual FWHM data, we thought that an attempt to determine a set of model parameters which better describe the data was warranted. The strong dependence of the FWHM on the circumferential slope errors, plus the fact of outdated metrology for this error term, suggested that a prudent and reasonable analysis would be to determine the average (i.e., the root-sum-square [RMS] of errors on P1 and H1) circumferential slope error which best fit the data. We did not carry out a fit in the formal sense to the FWHM scan data, but rather searched for that value with gave nearly the same FWHM as measured. Table 2 summarizes the cases run. Note that the slope errors in the table are given as the RSS of P1 and H1. In contrast to the sensitivity study done above, these cases include axial slope errors (Level II requirement), $\Delta \Delta R$ errors, sag, as well as the facility effects. The best fit corresponds to an average slope error of $3.0 \mu \mathrm{rad}$ per surface.

\begin{tabular}{|c|cc|}
\hline \multicolumn{3}{|c|}{ Table 2 } \\
Predicted & FWHM for Various Circumferential Slope Errors \\
\hline $\begin{array}{c}\text { RSS Error } \\
\mu \mathrm{rad}\end{array}$ & Y-FWHM & Z-FWHM \\
(arcsec) & $(\operatorname{arcsec})$ \\
\hline 0.0 & 0.209 & 0.208 \\
4.27 & 0.223 & 0.223 \\
5.80 & 0.234 & 0.238 \\
8.63 & 0.285 & 0.276 \\
11.68 & 0.326 & 0.321 \\
\hline
\end{tabular}

Figure 1 shows the comparison with the entire FWHM curve for the best fit circumferential slope errors. Note that a fit was not performed to this curve and only scaling in intensity was done. The agreement with the Y-scan data is remarkable. Differences with the Z-scan data (particularly the intensity jumps near \pm 50 $\mu \approx 1^{\prime \prime}$ ) are probably a result of residual gravitational distortions due to the bonded flexure pads which support the optics. At this time, neither the finite element mechanical model nor our raytrace model can faithfully reproduce such high frequency distortions in the optic.

\section{WING SCAN}

The wing scan data were originally intended to allow estimation of the amount of X-ray flux scattered beyond the largest diameter $(20 \mathrm{~mm})$ pinhole available in the VETA-I test and to provide a (hopefully small) 
correction to extrapolate to the effective area integrated over the whole focal plane. ${ }^{3}$ The test procedures called for stepping various size pinholes (from $3 \mathrm{~mm}$ diameter to $20 \mathrm{~mm}$ diameter) out to large offset angles from the beam center for various X-ray test energies. This data set has proved useful in characterizing the surface roughness of the optic as well as indicating the presence of surface particulate contamination.

Our approach was to assume that the observed surface brightness at large angles was a result of scattering from surface roughness. In this picture an in-surface spatial wavelength $1 / f$ diffracts (or scatters) light of a given wavelength $\lambda$ through an angle $\theta$ according to the the grating equation

$$
f=\frac{\theta \sin \alpha}{\lambda}
$$

with $\alpha$ as the mean grazing angle of the surface (here we use $51.2^{\prime}$ ). It is possible to relate ${ }^{4}$ the surface brightness $\psi(\theta)$ (at $\theta$ normalized to the total power in the focal plane) to the PSD of surface irregularities $W_{1}(f)$ through

$$
2 W_{1}(f)=\frac{f \psi(\theta) \lambda^{4}}{8 \pi(\sin \alpha)^{4}}
$$

The wing scan data were background subtracted and corrected for possible temporal variations in source intensity by dividing by the counting rate in the normalization detector. We used the wing scan data themselves to estimate the total flux for normalizing $\psi(\theta)$. We assumed azimuthal symmetry (which was an excellent approximation based on a comparison of data taken in the orthogonal Y- and Z-directions) and integrated the flux from the $3 \mathrm{~mm}$ diameter pinhole scan out to a radius of 2.036 milliradians ( $21 \mathrm{~mm}$ ). This was the only set of data which was available for nearly all the test energies and so this technique was used for consistency. The $\psi$ values also had corrections applied to compensate for the fact that we were sampling a steeply falling surface brightness distribution with a circular pinhole of finite size. In no case was a correction larger than $\sim 10 \%$ required. Figure 2 shows the wing scan data plotted in this representation.

Clearly the data in Fig. 2 are inconsistent with our main assumption, i.e., that all the scattering is due to surface roughness, since there is an apparent dependence of roughness with wavelength. For example, the inferred surface roughness from the carbon data alone $(\lambda=44 \AA)$ is $40 \AA$ per surface, while it is $11 \AA$ per surface from the molybdenum data $(\lambda=5.3 \AA)$. This (in addition to other evidence ${ }^{2}$ ) suggests the presence of particulate contamination on the mirrors. A simple three parameter model of scattering from dust on the surface has been developed ${ }^{2}$, combined with the surface scattering model, and fit to the data to yield the individual solid curves shown in Fig. 2 for each energy. We used a least-square minimization on the logarithmic data in lieu of a standard $\chi^{2}$ minimization using purely statistical errors. The fitted parameters of the dust model are consistent with other measurements of the surface contamination. ${ }^{2}$ In Fig. 2 the dashed curve shows the best-fit model for the surface PSD; the numerical values of this fit are $\sigma=7.3 \pm 0.6 \AA$ (per surface) and $p=1.22 \pm 0.13$ (90\% confidence errors for a single parameter).

Figure 4 shows two-dimensional contours for the amplitude and index of the power-law PSD from fits to the wing scan data (the dashed set of contours on the right side). The errors on the data used to produce this figure were estimated from the root-sum-square of the logarithmic residuals from the best-fit model (shown in the bottom panel of Fig. 2).

\section{ENCIRCLED ENERGY}

The encircled energy data were processed in detail to remove numerous test effects. These included background subtraction, pulse pileup, and deadtime, as well as corrections to remove the known spectral impurity of the incident $\mathrm{X}$-ray spectrum, due mainly to continuum emission from electron bremsstrahlung in the X-ray target. ${ }^{5}$ Additional data reduction was implemented to remove the effects of the wire mesh 

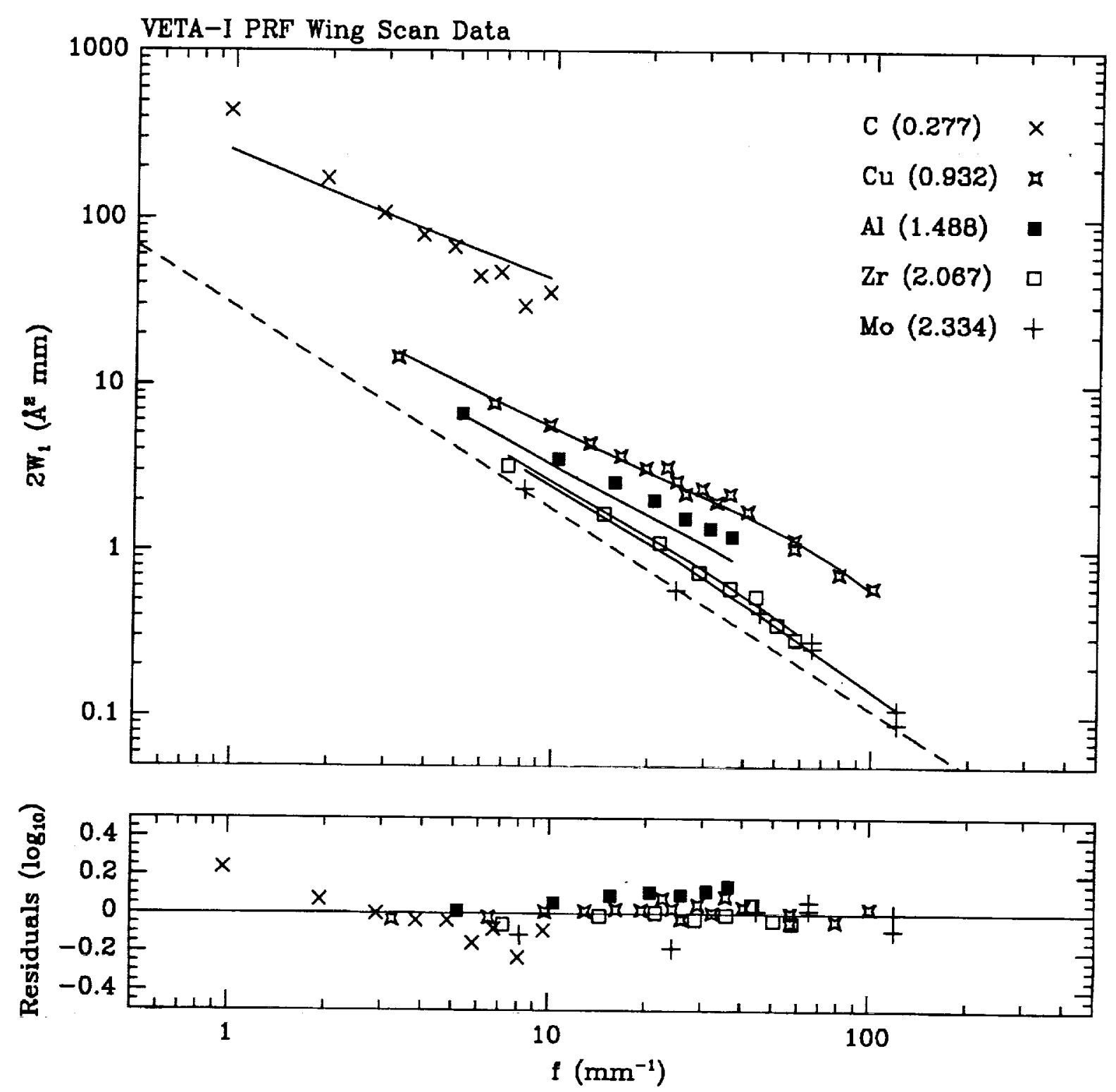

Figure 2 - Large angle scattering (wing scan) data from the five $\mathrm{X}$-ray test energies scaled and plotted in terms of the PSD $\left(2 W_{1}\right)$ of surface irregularities vs. spatial frequency $f$. The dashed curve indicates what would be expected if only scattering from a rough surface were involved. The evident differences between the several test energies strongly indicate the presence of particulate contamination on the surface. The individual solid curves show the best-fit model for scattering from both surface roughness and particulate contamination for the various test energies. The lower panel shows the logarithmic residuals between the data and best-fit model.

supporting the thin polypropylene window of the low energy proportional counters used in the test. ${ }^{6}$ These processing steps allowed us to reduce the average fractional error on our encircled energy data from $3.8 \%$ to $0.9 \%$. 

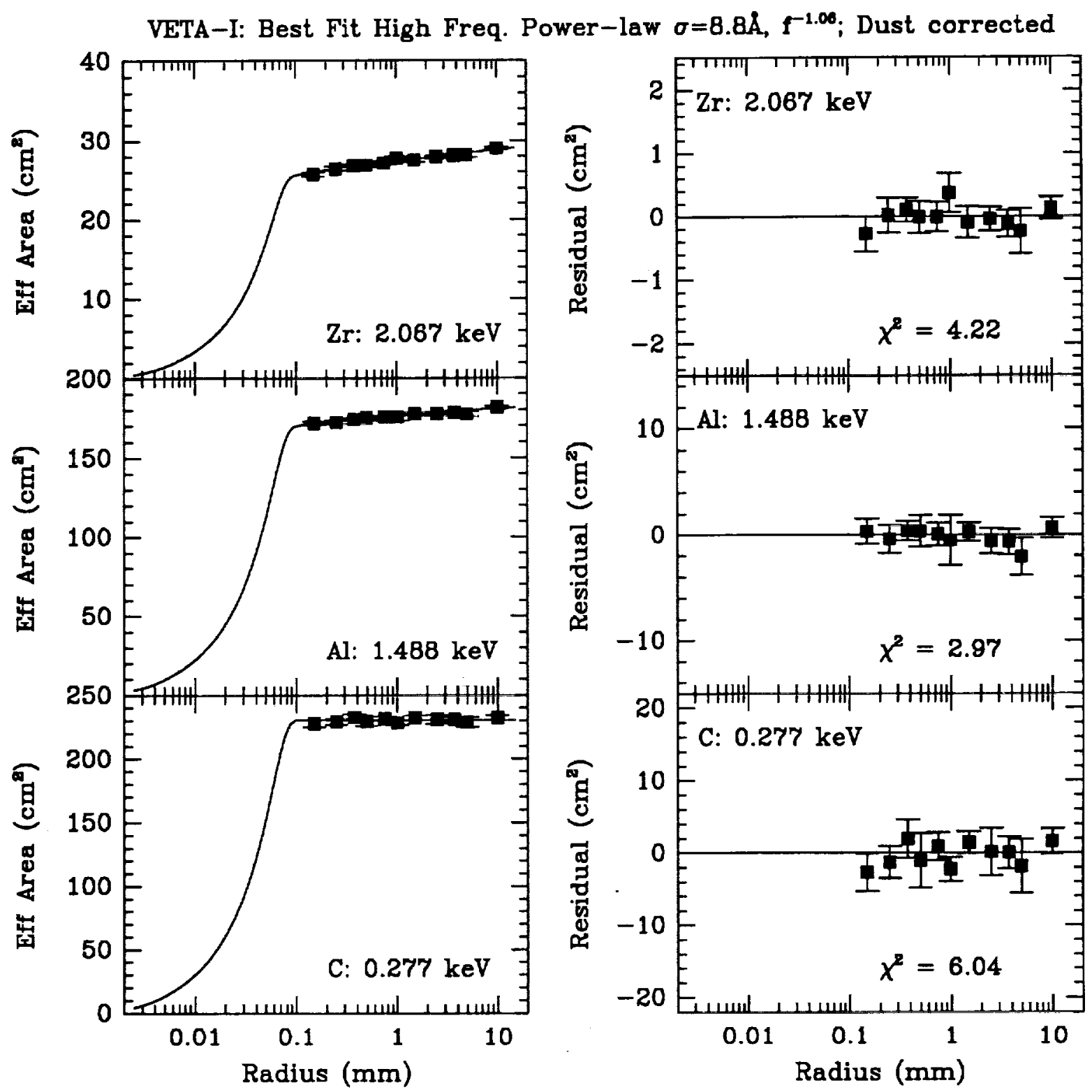

Figure 3 - Encircled energy data for three X-ray test energies. The panels on the left show the data and the best-fit raytrace model; the panels on the right show the residuals.

Figure 3 presents the encircled energy data at three test energies (labelled by the material composition of the anode target) for which it was possible to carry out the complete processing steps outlined above. The data are given in effective area units by referencing the number of X-ray events observed through each pinhole by the focal plane proportional counter to the corresponding numer of counts observed in the (nominally identical) normalization proportional counter positioned at the entrance aperture of the VETA-I.

We compare the encircled energy data at three $\mathrm{X}$-ray test energies with our raytrace model. Note that the results in this section include the updated value for the circumferential slope errors of $3.0 \mu \mathrm{rad}$ per 
Fits to Power-Law PSD

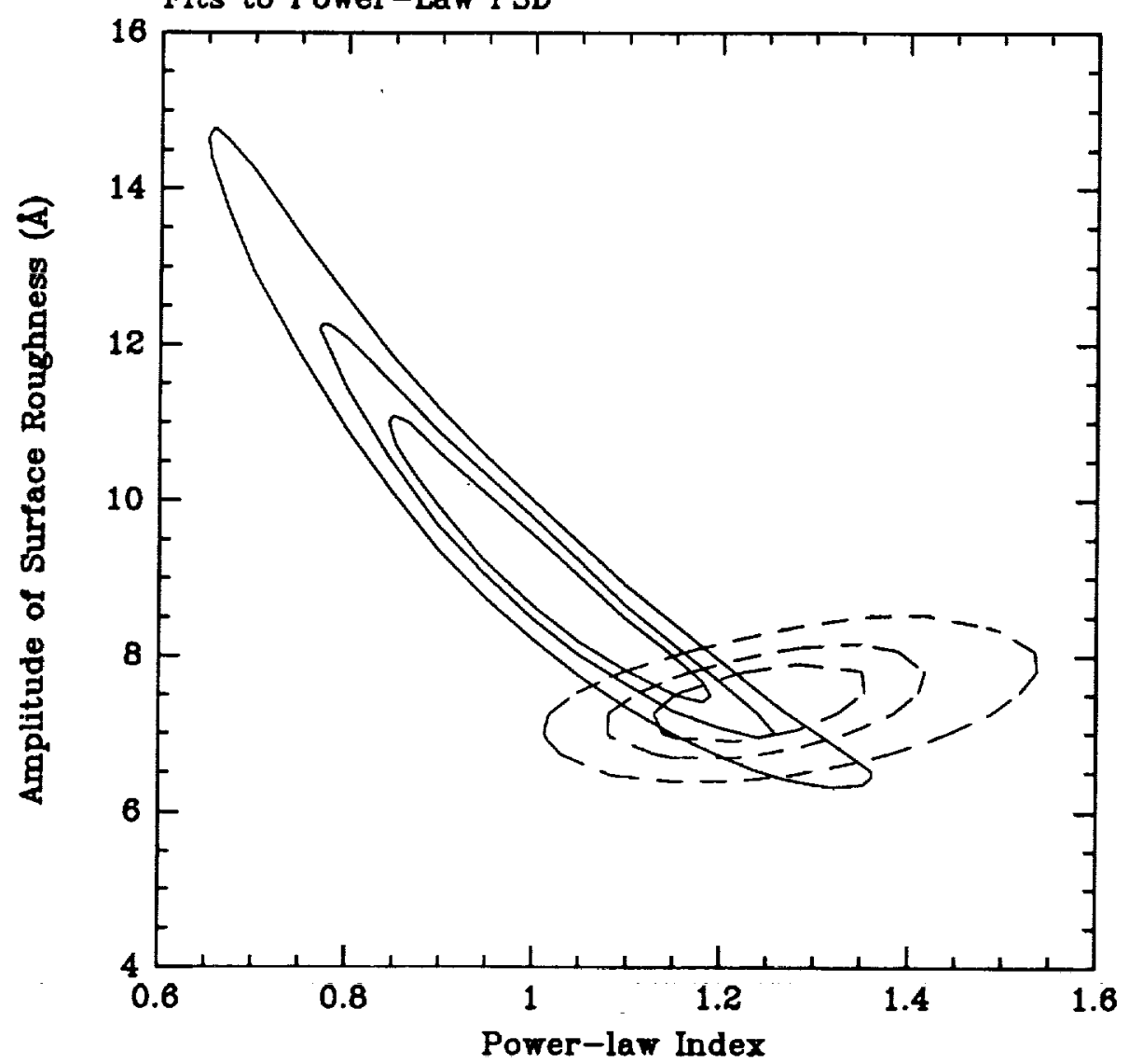

Figure 4 - Contours of constant $\chi^{2}$ for fits of a power-law PSD for surface roughness to the wing scan data (dashed contours) and encircled energy data (solid contours). We show the $68 \%, 90 \%$, and $99 \%$ confidence levels corresponding to 2 interesting free parameters: the power-law index (abscissa) and the amplitude of surface roughness (ordinate).

surface from the FWHM analysis presented in $\S 3$. We also incorporate the best-fit dust scattering model from the wing scan fits discussed in $\$ 4$. The effect of dust scattering on the EE data is weak: from the smallest pinhole considered $(0.3 \mathrm{~mm}$ diameter) to the largest (20 mm diameter) there is only a $2 \%$ difference in the encircled energy due to scattering from particulate contamination.

Some comments about how the model and data were normalized are needed. At the present time we are not able to accurately estimate the amount of X-ray flux scattered by the mirror beyond the largest pinhole used in these measurements and thus we do not know the total power (or transmitted energy) in the focal plane. As mentioned above, the data are given in effective area units, while the raytrace produces encircled energy models with values between zero and unity. In our model comparisons we scale the model to the data by determining the multiplicative normalization factor which minimizes $\chi^{2}$. This is done separately for the data at each $\mathrm{X}$-ray energy. The multiplicative factor is the total effective area (integrated over the focal plane) at that energy.

In order to fit for the parameters of the power-law PSD we generated a large grid of model encircled energy distributions over a range of amplitudes and power-law indices. For each amplitude-index pair, three raytraces were run corresponding to each of the three X-ray test energies. At each point in this twodimensional parameter space the data and models were compared (for each test energy separately) and a 

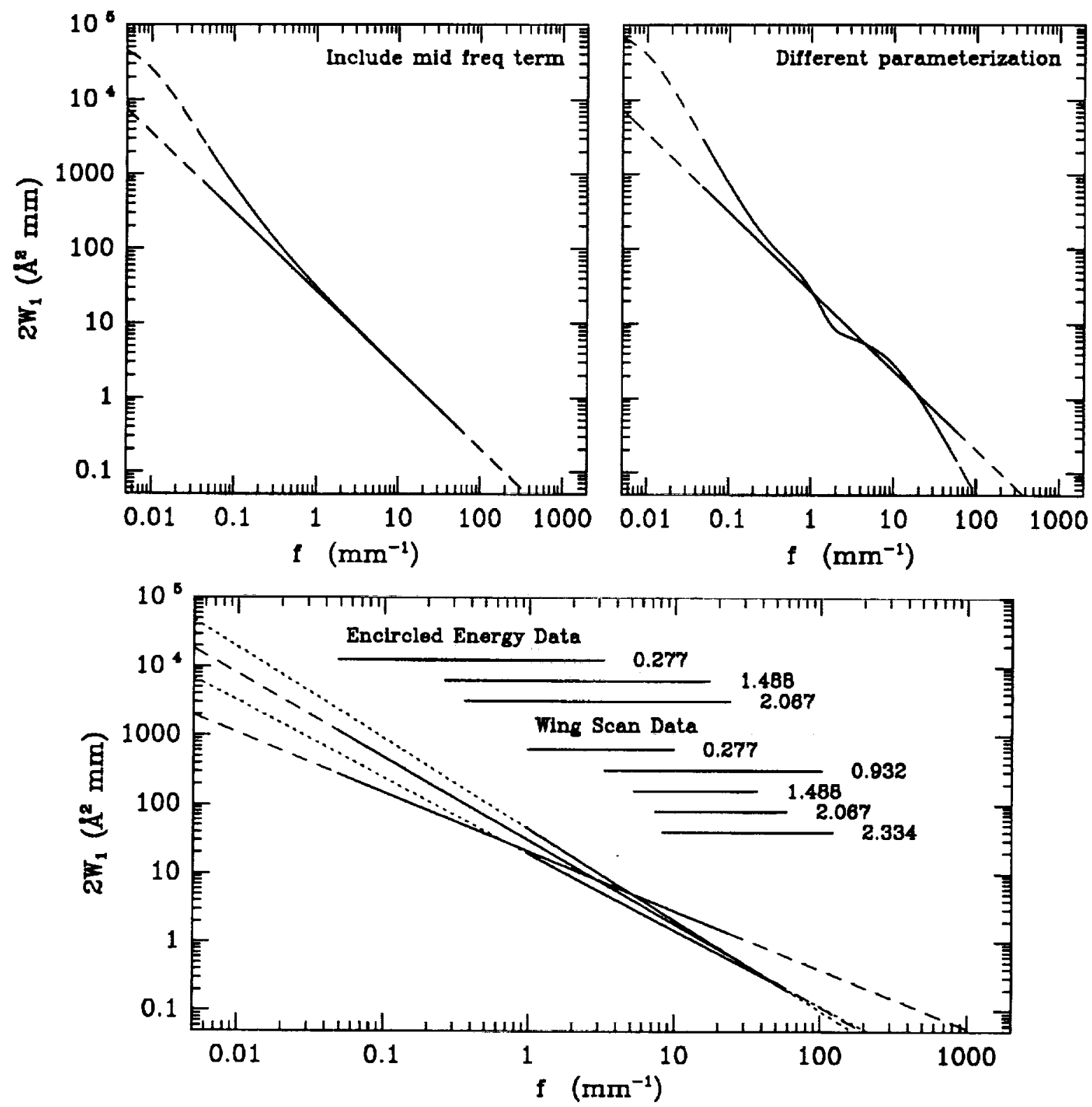

Figure 5 - Estimated PSD of the polished optical surface of the VETA-I based on the X-ray test measurements. The top two panels show two possible variations on the allowed shape of the PSD. The bottom panel shows the allowed range of surface PSD from fits of a pure power-law to the encircled energy and wing scan data. The range in $f$-values which was sampled by each data set is indicated in this panel. Extrapolations of the PSD beyond these ranges appear as dashed lines. 
total $\chi^{2}$ value for that point was determined. The best fit from these data occurred for $\sigma=8.8_{-1.4}^{+2.2} \AA$ and $p=1.06_{-0.20}^{+0.15}$ with a grand combined $\chi^{2}=13.2$ for 28 d.o.f. The smooth curves shown in the left panels of Fig. 3 correspond to this best fit and the residuals are in the right panels. The fit for each of the test energies is good, as indicated by the individual $\chi^{2}$ values labelling the panels.

The two-dimensional $\chi^{2}$ contours for fits to the encircled energy data are shown in Fig. 4 as the solid contours toward the left side. These are consistent, even at the $68 \%$ confidence level, with the wing scan data results.

\section{CONCLUSIONS}

We have used X-ray measurements of the VETA-I to estimate the surface finish quality of the largest pair of AXAF mirror elements. We find that the narrow FWHM of the PRF requires that the circumferential slope errors be approximately $3.0 \mu \mathrm{rad}$ per surface. Both the wing scan and encircled energy data can be well fit by a PSD for the surface roughness which falls as a power-law in spatial frequency with index 1.2 and amplitude $\sigma \approx 7.5 \AA$. The wing scan data also reveal evidence for a significant level of particulate contamination on the VETA-I mirror.

The X-ray data are unable to make strong statements about the actual detailed functional dependence of the surface PSD on spatial frequency. For example the data allow the inclusion of a mid-spatial frequency term (with a correlation length of $18 \mathrm{~mm}$ ) at a level of about $20 \AA$, as shown in the top left panel of Fig. 5 . An entirely different parameterization of the surface PSD (based on the set of terms used to derive specifications on HRMA performance), also provides an acceptable fit to the X-ray data (see the top right panel of Fig. 5). The lower panel of the same figure shows the range of pure power-law PSD models allowed by the encircled energy data and the wing scan data. Although it is not possible to exclude a single power-law model as a description of the data, there may be some indication of a steepening of the PSD with increasing spatial frequency. We look forward to the final optical metrology data of the VETA-I, which will allow us to investigate these and other models characterizing the surface finish quality of the VETA-I.

This research was supported in part by NASA contract NAS8-36123.

\section{REFERENCES}

1. P. Zhao, E. M. Kellogg, D. A. Schwartz, M. A. Fulton, and C. Bower, these proceedings, 1992.

2. S. L. O'Dell, R. F. Elsner, J. J. Kolodziejczak, M. C. Weisskopf, J. P. Hughes, and L. Van Speybroeck, these proceedings, 1992.

3. E. M. Kellogg, G. Chartas, J. P. Hughes, L. Van Speybroeck, P. Zhao, M. C. Weisskopf, R. F. Elsner, and S. L. O'Dell, these proceedings, 1992.

4. P. Beckmann, and A. Spizzichino, The Scattering of Electromagnetic Waves from Rough Surfaces, (Oxford: Pergamon), 1963.

5. G. Chartas, K. Flanagan, J. P. Hughes, E. M. Kellogg, D. Nguyen, M. Zombeck, M. Joy, and J. Kolodziejczak, these proceedings, 1992.

6. P. Zhao, M. D. Freeman, J. P. Hughes, E. M. Kellogg, D. T. Nguyen, M. Joy, and J. J. Kolodziejczak, these proceedings, 1992. 